\title{
Controlling the Tektronix 4006 graphics terminal with OS/8 BASIC
}

\author{
JAMES H. HOWARD, JR. \\ Catholic University of America, Washington, D. C. 20064
}

\begin{abstract}
Several BASIC-callable assembler subroutines are described that permit relatively straightforward interaction with a Tektronix $\mathbf{4 0 0 6}$ or equivalent graphics terminal. These routines enable input, alphanumeric output, and graphic output with or without automatic coordinate transformation. The routines can be used for the graphic presentation of data or, with additional laboratory routines, for stimulus presentation in psychological experiments.
\end{abstract}

This paper describes a series of BASIC-callable assembler routines designed to control a Tektronix 4006 graphics terminal using a PDP-8/e computer with the OS/8 operating system. This terminal provides a highresolution $(1,024$ by 780 visible points) storage-tube graphics capability that can solve a number of problems encountered in psychological research (Tektronix, 1978). Its low cost (less than $\$ 3,000$ ) and simple interface requirements (KL8-E asynchronous interface) have made the 4006 terminal popular in this application. The subroutines described here can be useful for either the on-line presentation of simple visual stimuli in psychological experiments or the off-line display of experimental data.

In our laboratory, the Tektronix terminal has been used primarily to present stimuli in psychophysical and visual memory studies. Accordingly, the software was coded to be compatible with a number of generalpurpose timing and control utilities that were described in a previous paper (Howard, 1979). These utilities, together with the graphics subroutines presented here, have enabled us to control a variety of experiments with little difficulty. Table 1 presents an overview of the software, including an OS/8 BASIC function mnemonic, a calling sequence, a BASIC run-time system entry point, and a brief description for each subroutine. The first five functions presented in Table 1 are taken from the previously described laboratory package and will not be discussed in detail here.

Although our application has focused on the on-line presentation of experimental stimuli, the present routines (Functions 6-12 in Table 1) may also be used for the graphic display of data. To accomplish this, the user would have to write a BASIC program to perform

This work was supported in part by grants from the U.S. Army Research Institute and the National Institutes of Mental Health to the Catholic University of America. The author thanks Jim Ballas, Darlene Howard, Cyril Simone, and an anonymous reviewer for their comments on an earlier draft of this manuscript. Requests for reprints or copies of source listings may be addressed to James H. Howard, Jr., Human Performance Laboratory, The Catholic University, Washington, D.C. 20064. data scaling, axis labeling, and whatever other capabilities that may be required. Interaction with the graphics terminal, as well as simple data scaling and transformations, can be handled by calls to the present routines.

The graphic control functions described here require only a minimal OS/8 system, OS/8 BASIC, and a Tektronix 4006,4010 , or compatible terminal. ${ }^{1}$ If the functions are to be used for experimental control (Functions 1-5 in Table 1), then a DK8-EC real-time clock and a DR8-EA digital input/output interface must also be available on the system. The user who is not interested in on-line experimental control may alternatively purchase the more extensive PLOT-10 FORTRAN IV subroutine package from Tektronix. However, this alternative requires an additional software investment of as much as $\$ 3,400$ and must be modified to run on even large-memory (32K words core) PDP-8/e systems.

\section{FUNCTION DEFINITIONS}

The first three functions, ALP, ALC, and TIN, enable the user to interact with the Tektronix terminal in an alphanumeric mode. Text may be displayed on the screen or received from the terminal keyboard. These functions have been adapted from similar routines designed to interact with a CRT terminal (Howard, 1979). The MOV and DRW routines interact with the terminal in graphic mode, plotting points or lines as specified by coordinate arguments. The SET routine provides the added capability of performing a transformation of graphic point coordinate arguments automatically before plotting takes place. Finally, the CLR routine enables the user to erase the screen.

\section{ALP}

This function is used to display character strings on the screen. The statement $Z=A L P(S \$)$ will cause the terminal to enter the alphanumeric mode and the string $S \$$ to be displayed beginning at the current cursor address. The cursor address may be reset to the upper left corner of the screen with a CLR call, or it may be 
Table 1

Mnemonic, Calling Syntax, and Entry Point for Each Laboratory Routine

\begin{tabular}{clcl}
\hline Function & Calling Syntax & Entry Point & \multicolumn{1}{c}{ Description } \\
\hline 1. DEL & $\mathrm{Z}=\mathrm{DEL}(\mathrm{N})$ & 3400 & Delay N msec \\
2. DRS & $\mathrm{Z}=\mathrm{DRS}(\mathrm{N})$ & 3445 & Set output bit(s) \\
3. DRC & $\mathrm{Z}=\mathrm{DRC}(\mathrm{N})$ & 3451 & Clear output bit(s) \\
4. DRI & $\mathrm{R}=\mathrm{DRI}(\mathrm{N})$ & 3530 & Read input bit(s) \\
5. GRT & $\mathrm{T}=\mathrm{GRT}(\mathrm{Z})$ & 3741 & Get reaction time for DRI \\
6. ALP & $\mathrm{Z}=\mathrm{ALP}(\mathrm{S} \$)$ & 3455 & Output alphanumeric string \\
7. ALC & $\mathrm{Z}=\mathrm{ALC}(\mathrm{S} \$)$ & 3521 & Output alphanumeric control string \\
8. TIN & $\mathrm{T}=\mathrm{TIN}(\mathrm{F} \$, N)$ & 3600 & Read string from keyboard \\
9. MOV & $\mathrm{Z}=\mathrm{MOV}(\mathrm{X}, \mathrm{Y})$ & 4024 & Move cursor to screen coordinates $\mathrm{X}, \mathrm{Y}$ \\
10. DRW & $\mathrm{Z}=\mathrm{DRW}(\mathrm{X}, \mathrm{Y})$ & 4057 & Draw vector to screen coordinates $X, Y$ \\
11.SET & $\mathrm{Z}=\mathrm{SET}(\mathrm{A}, \mathrm{B})$ & 4316 & Set translation constant A to B \\
12. CLR & $\mathrm{Z}=\mathrm{CLR}(\mathrm{X})$ & Erase screen \\
\hline
\end{tabular}

specified by a preliminary MOV function call. String length is restricted to 72 characters/call by OS/8 BASIC, and the presentation rate is limited to 4,800 baud by the Tektronix terminal. Longer strings may be displayed successively by repeated calls to the ALP function.

\section{ALC}

Since OS/8 BASIC uses a 6 -bit internal code for character strings, ASCII control characters (i.e., Bit 7 suppressed) cannot be displayed using the ALP function. ALC solves this problem by enabling the user to output a string of control characters. The statement $Z=A L C$ ("GGGG") would first cause the terminal to enter the alphanumeric mode and then transmit a series of four ASCII control Gs. As on most standard terminals, a control $G$ rings the bell on the Tektronix terminal.

\section{TIN}

This function enables the user to read character strings from the Tektronix keyboard in a number of formats. The function is called by a $T=\operatorname{TIN}(F \$, N)$ statement. The F\$ argument specifies whether the input characters should be echoed at the current cursor position on the display as they are entered. An $\mathrm{F} \$=$ "ECHO" specifies the echo mode, whereas $\mathrm{F} \$=$ "NOECHO" specifies the no-echo mode. A preliminary call to the MOV function may be required to have the characters echoed at a specific screen location.

The numeric argument, $\mathrm{N}$, specifies the total number of characters to be entered. Fewer than $\mathrm{N}$ characters will be read if either (1) a carriage return is entered before $\mathrm{N}$ characters are read or (2) the input string has been dimensioned to be smaller than N. In the latter case, TIN will read only as many characters as can be stored in the input string, up to the OS/8 BASIC limit of 72 characters. Characters are always read into the first string dimensioned (implicitly or explicitly) in the BASIC program. An explanation of this unusual convention may be found in Howard $(1979$, p. 439). Upon return, the $\mathrm{T}$ variable will contain the response latency timed from the function call to the first character input, accurate to $1 \mathrm{msec}$.

The following program segment illustrates how the first three functions are used. First, a character string is displayed on the screen, warning that a response is required when the bell rings. After a delay of $1 \mathrm{sec}$ $(1,000 \mathrm{msec})$, the terminal beeps and a single character is read from the keyboard. Finally, the response character and input latency are printed on the system console. In this example, $R \$$ constitutes the input string, since it is the first string dimensioned in the program and the input character is not echoed as it is entered.

$$
\begin{aligned}
& 5 \text { DIM R\$(2), A\$(72) } \\
& 100 \text { A } \$ \text { = "PLEASE RESPOND } \\
& \text { WHEN THE BELL RINGS" } \\
& 110 \mathrm{Z}=\mathrm{ALP}(\mathrm{A} \$) \\
& 120 \mathrm{Z}=\mathrm{DEL}(1000) \\
& 130 \mathrm{Z}=\mathrm{ALP}(\text { ' } \mathrm{G} \text { ") } \\
& 140 \mathrm{~T}=\text { TIN("NOECHO", 1) } \\
& 150 \text { PRINT "RESPONSE WAS" } \\
& \text {,RS, "WITH LATENCY";T; } \\
& \text { "MSEC" }
\end{aligned}
$$

Since the ALP, ALC, and TIN functions parallel the CRO, CRC, and CRI functions described in Howard (1979), additional illustrations can be found in that source.

\section{MOV}

This function causes the terminal to enter the graphic mode and draw an "invisible" vector from the present cursor position to a specified location. The CRT beam is not energized during the positioning move. The desired location is specified by the $(X, Y)$ coordinates in the function call $Z=\operatorname{MOV}(X, Y)$. A call to this routine can serve either to specify the location of alphanumeric output when it is followed by an ALP function call or to initialize the terminal for graphic output when followed by a DRW function call.

When using absolute screen addressing (see SET function description), the $\mathrm{X}$ and $\mathrm{Y}$ coordinates shculd be integers and must be within the range $0-1,023$. The 
lower left corner of the screen is designated $(0,0)$, whereas the upper right corner is $(1023,780)$. Although a full 10-bit (1,024-point) range may be specified along both dimensions, only 780 points are actually visible on the Tektronix screen along the vertical, or $Y$, dimension. Consequently, the statement $Z=\operatorname{MOV}(512,390)$ would position the beam in the approximate center of the screen.

\section{DRW}

This function enables the user to draw a vector from the present cursor position to a specified endpoint. It must always be preceded by a MOV or another DRW function call. In absolute addressing, the screen coordinates are determined as described previously for the MOV function. To illustrate the use of this function, the following program segment draws a square in the lower left corner of the screen. The resulting square will be 200 Tektronix screen units on each side, approximately $20 \%$ of the horizontal dimension and $26 \%$ of the vertical dimension.

$$
\begin{aligned}
100 Z & =\operatorname{MOV}(0,0) \\
110 Z & =\operatorname{DRW}(200,0) \\
120 Z & =\operatorname{DRW}(200,200) \\
130 Z & =\operatorname{DRW}(0,200) \\
140 Z & =\operatorname{DRW}(0,0)
\end{aligned}
$$

The following example plots two adjacent squares of equal size, one in the lower left corner and the other 400 screen units farther to the right.

$$
\begin{aligned}
& 100 \mathrm{Y}=0 \\
& 110 \mathrm{Y} 1=200 \\
& 120 \mathrm{FOR} \mathrm{I}=0 \text { TO } 400 \text { STEP } 400 \\
& 130 \mathrm{X}=0+\mathrm{I} \\
& 140 \mathrm{X} 1=200+\mathrm{I} \\
& 150 \mathrm{Z}=\mathrm{MOV}(\mathrm{X}, \mathrm{Y}) \\
& 160 \mathrm{Z}=\mathrm{DRW}(\mathrm{X} 1, \mathrm{Y}) \\
& 170 \mathrm{Z}=\mathrm{DRW}(\mathrm{X} 1, \mathrm{Y} 1) \\
& 180 \mathrm{Z}=\mathrm{DRW}(\mathrm{X}, \mathrm{Y} 1) \\
& 190 \mathrm{Z}=\mathrm{DRW}(\mathrm{X}, \mathrm{Y}) \\
& 200 \mathrm{NEXT} \mathrm{I}
\end{aligned}
$$

\section{SET}

The SET function enhances graphic addressing by enabling automatic coordinate transformations. Once a transformation is specified by a series of calls to this function, all subsequent MOV and DRW calls will utilize transformed rather than absolute coordinates. Since the coordinates specified in a MOV or DRW function call should be integers within the $0-1,023$ range, transformations implemented with the SET function can simplify graphic plotting greatly when either negative or noninteger coordinate values are used. Ordinarily, the user would have to transform such values to Tektronix absolute screen coordinates explicitly before plotting.
The SET function enables the user to accomplish this implicitly with minimal programming effort.

Transformations are specified by setting eight transformation constants (C1-C8) using the SET function. The statement $Z=$ SET $(A, B)$ will set transformation constant $A$ to a value of $B(i . e ., C A=B$ ). The first four constants ( $\mathrm{Cl}-\mathrm{C} 4)$ may be used to establish simple axis scaling and translation. This is designed to deal with negative values or coordinates that are either very small or very large. The transformed coordinates that result $\left(X^{\prime}, Y^{\prime}\right)$ are simple linear functions of the coordinates input to the MOV and DRW functions $(\mathrm{X}, \mathrm{Y}) ; \mathrm{X}^{\prime}=$ $\mathrm{C} 1 \mathrm{X}+\mathrm{C} 2, \mathrm{Y}^{\prime}=\mathrm{C} 3 \mathrm{Y}+\mathrm{C} 4$, where the constants may be specified by successive calls to the SET function. Setting $\mathrm{C} 2$ and $\mathrm{C} 4$ to 512 and 390, respectively, enables the user to work with $X$ coordinates between -512 and +511 and $Y$ coordinates between -390 and +389 . Similarly, data ranging between -2.0 and +2.0 could be plotted easily using the MOV and DRW functions if $\mathrm{C} 1$ and $\mathrm{C} 3$ were set to 256 and 195 , respectively.

The last four transformation constants (C5-C8) allow more complex transformations to be specified. In particular, these transformation constants may be thought of as cells in a transformation matrix. The transformed coordinates $\left(\mathrm{X}^{\prime} \mathrm{Y}^{\prime}\right)$ are determined by the product of this matrix and the input coordinate vector $(\mathrm{X}, \mathrm{Y})$ :

$$
\left(\begin{array}{l}
X^{\prime} \\
Y^{\prime}
\end{array}\right)=\left(\begin{array}{ll}
\mathrm{C} 5 & \mathrm{C} 6 \\
\mathrm{C} 7 & \mathrm{C} 8
\end{array}\right)\left(\begin{array}{l}
\mathrm{X} \\
\mathrm{Y}
\end{array}\right)
$$

This matrix transformation affords considerable flexibility, since any general linear transformation, including axis rotation, can be specified easily. For example, if an automatic clockwise rotation of $\theta \mathrm{rad}$ is desired, then C5 and $\mathrm{C} 8$ should be set to $\operatorname{Cos}(\theta), \mathrm{C} 6$ to $\operatorname{Sin}(\theta)$, and C7 to $-\operatorname{Sin}(\theta)$ (Green \& Carroll, 1976). Once these constants have been set, data plotted using MOV and DRW will be rotated through $\theta$ rad on the Tektronix screen.

The coordinate transformations are computed using assembler calls to the BASIC floating-point software. Although some of these computations are completed while waiting for the transmit ready flag (graphic operations on the Tektronix require the output of multiple ASCII characters), a slightly slower output rate results with coordinate transformations. To minimize these delays, no default transformations are computed if the SET function has not been called. Furthermore, the transformation constants may be set or altered individually. For convenience, the parameters are initialized to translate the graphic origin to the center of the screen without any scaling or rotation. Consequently, to implement this transformation, it is necessary only to set any one of the first four constants $(\mathrm{C} 1-\mathrm{C} 4)$ to its proper value; the other constants will assume their default values $(\mathrm{C} 1=1, \mathrm{C} 2=512, \mathrm{C} 3=1, \mathrm{C} 4=390)$. 
Table 2

Sample Program to Plot a Square 200 Screen Units per Side, Centered on Screen, and Rotated Through .78 Rad (45 Deg)

95 REM SET UP TRANSFORMATION CONSTANTS

100 FOR I=1 TO 4

110 READ J

120 Z $=\operatorname{SET}(\mathbf{I}, \mathbf{J})$

130 NEXT I

140 DATA 400,512,400,390

$150 \mathrm{C}=\mathrm{COS}(.78)$

$160 \quad \mathrm{~S}=\operatorname{SIN}(.78)$

$170 \quad Z=S E T(5, C)$

$180 \quad Z=\operatorname{SET}(6, S)$

$190 \quad Z=\operatorname{SET}(7,-S)$

$200 \quad \mathrm{Z}=\mathrm{SET}(8, \mathrm{C})$

205 REM NOW PLOT SQUARE

$210 \mathrm{Z}=\operatorname{MOV}(-100,-100)$

$220 \quad Z=D R W(-100,100)$

$230 \quad Z=D R W(100,100)$

$240 \quad Z=D R W(100,-100)$

$250 \quad Z=$ DRW $(-100,-100)$

Similarly, the matrix transformation constants will take on their default values if any one of them is set (C5 $=1$, $\mathrm{C} 6=0, \mathrm{C} 7=0, \mathrm{C} 8=1$ ). Calling the SET routine with a constant value of 0 will clear all transformations and cause a return to the absolute coordinate system [i.e., $\mathrm{Z}=\operatorname{SET}(0,0)]$.

The program segment in Table 2 illustrates use of the SET function. Execution of this program results in the plot of Figure 1a, a square 200 screen units per side, centered on the screen and rotated through $.78 \mathrm{rad}$ (45 deg) ${ }^{2}$

\section{CLR}

The clear function enables the user to erase the screen. Only a dummy argument is required for this function: $Z=C L R(Z)$. Since the Tektronix terminal uses a conventional storage tube, the CLR function causes the entire screen to be energized. A delay of approximately $800 \mathrm{msec}$ is required for the phosphor to stabilize after this flash before additional data can be output (see Footnote 1).

\section{EXAMPLE PROGRAM}

The program presented in Table 3 illustrates how the functions may be used to plot the simple figures shown in Figure 1b. The squares on the left are of different areas, whereas those on the right are of equal areas but at different angles of rotation.

Each user function must be defined by a UDEF statement (Statements 20 and 30 ) to provide the BASIC compiler with information regarding the proper number and type of arguments for each function (Digital Equipment Corporation, 1974, chap. 6). The program also contains an explicit dimension statement (Statement 40) to allocate storage for string input from the TIN function.

The program consists of three major parts, terminal input and two output loops, one for each set of squares. The input segment (Statements 50-80) erases the Tektronix screen and displays a message indicating that an "S" should be entered to begin output. The message will appear in the upper left portion of the screen, since CLR resets the cursor to this position. The program then waits for an " $S$ " to be entered on the keyboard. Input characters will not be echoed as they are entered. Once an "S" is received, the ALC function is used to ring the bell, the start message is erased, and graphic output begins. At this point, the latency between the TIN function call and the keyboard input, accurate to $1 \mathrm{msec}$, is available in variable $\mathrm{T}$.

A SET function call precedes the first graphic output loop to set the transformed origin for plotting to the center of the left half of the screen. Statement 90 sets the translation constant $\mathrm{C} 2$ for the horizontal axis to 256 , one quarter of the full-scale range. The remaining transformation constants will assume their default values (no horizontal and vertical scaling, $\mathrm{C} 1$ and $\mathrm{C} 3=$ 1.0; $\mathrm{Y}$ coordinate translation to one-half the vertical range, $\mathrm{C} 4=390$ ). The output loop in Statements 100 170 plots 10 squares ranging in size from 40 screen units per side when $I$ is 1 to 220 units per side when $I$ is 5.5 .
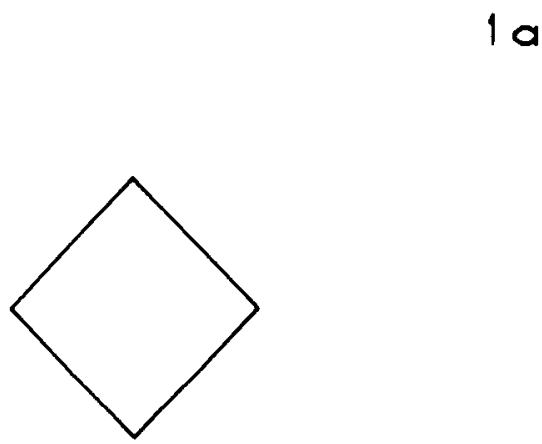

$1 b$
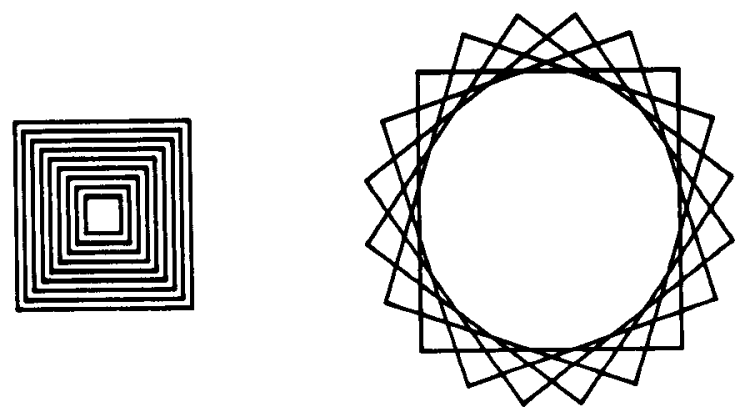

Figure 1. Graphic output produced by sample programs. Figure la shows rotated square produced by program in Table 2 , and Figure $1 b$ shows sets of squares plotted by program in Table 3. 
Table 3

Sample Program to Illustrate Use of Tektronix Functions

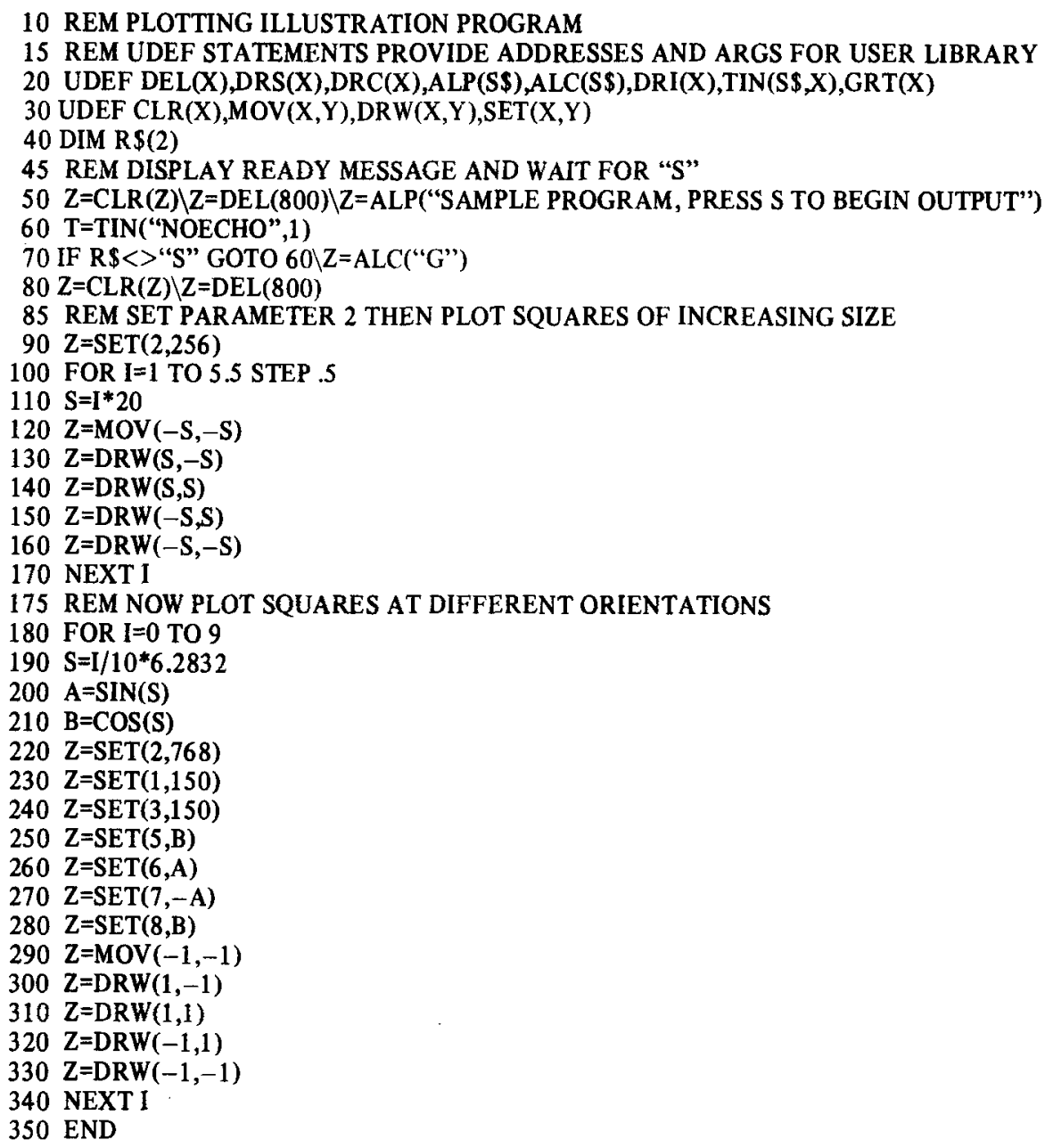

The square is specified by positive and negative coordinates of one-half the desired width, since automatic axis translation was enabled by the SET function call.

The second output loop, Statements 180-340, plots 10 squares of equal size at different angles of orientation. Three SET statements are required: one to specify a horizontal translation of 768 (Statement 220) and two to scale the horizontal and vertical axes, respectively (Statements 230 and 240). Since the vertical translation constant, $\mathrm{C} 4$, has not been altered, it will remain at its default value of 390 . The transformed origin is now centered in the right half of the screen. Note that in this case, the set calls must be included inside the loop because the BASIC arithmetic function overlay is required for the SIN and COS functions in Statements 200 and 210 . The arithmetic overlay will be loaded dynamically during program execution, thereby destroying the user overlay. The rotations are achieved by setting the matrix transformation constants (C5-C8) in Statements 250-280. Constants C5 and C8 are set to the cosine of the desired angle, $\mathrm{C} 6$ to the sine, and $\mathrm{C} 7$ to the negative sine. The square is plotted by specifying positive and negative unit coordinates, since automatic scaling was enabled for both axes. Multiple squares of 300 screen units per side are plotted at different orientations, since the rotation transformation is reinitialized on each pass through the loop. The angle of rotation varies from $.00 \mathrm{rad}(0 \mathrm{deg})$ to $5.56 \mathrm{rad}(324 \mathrm{deg})$ in equal steps of $.63 \mathrm{rad}(36 \mathrm{deg})$.

\section{SUMMARY}

The software described in this paper has been in regular use in our laboratory for approximately 2 years. We have found it to be an effective way to handle the otherwise awkward programming required to display information on the Tektronix graphics terminal. The OS/8 BASIC user functions are relatively easy to learn and do not require an in-depth understanding of the Tektronix control sequences. The software would also be useful for others who may wish to code their own routines for special-purpose graphics operations. Further 
information for those individuals may be found in Digital Equipment Corporation (1974) and Tektronix (1978).

\section{NOTES}

1. The Tektronix 4000 series $(4006,4010)$ terminals use storage-tube graphics technology and may be limited for many applications. Alphanumeric data presentation is restricted to uppercase characters presented and displayed at a 4,800-baud rate. Since a conventional storage CRT is used, screen erasure involves energizing the entire screen. This produces a relatively bright flash that can have an unwanted masking effect and leaves the phosphor unstable for approximately $800 \mathrm{msec}$. These limitations are avoided with the similarly priced (approximately $\$ 3,200$ ) raster-scan CRT terminal distributed by Digital Engineering Corporation. This terminal has full upper- and lowercase alphanumeric capability, high-resolution graphics ( 640 by 480 ), 19,200 -baud presentation rates, and nonmasking partial or whole screen erase. Although this terminal is actually a modified Digital Equipment Corporation VT-100, it responds to the
Tektronix 4000 series graphics protocols and may be used with the present software package.

2. Figures $1 \mathrm{a}$ and $1 \mathrm{~b}$ were plotted using the present software and a Tektronix 4662 digital plotter. Although more sophisticated than the 4006 terminal, the plotter is also compatible with graphic output protocols produces by the present software.

\section{REFERENCES}

Digital Equipment Corporation. OS/8 handbook. Maynard, Mass: Author, 1974.

Green, P. E., \& Carroll, J. D. Mathematical tools for applied multivariate analysis. New York: Academic Press, 1976.

Howard, J. H., JR. A multipurpose laboratory package for OS/8 BASIC. Behavior Research Methods \& Instrumentation, $1979,11,437-444$.

Tektronix. 4006-1 computer display terminal users manual. Beaverton, Ore: Author, 1978.

(Received for publication February 13, 1981; revision accepted April 18, 1981.) 